\title{
Efficacy of Trunk Rehabilitation and Balance Training On Trunk Control, Balance and Gait in Post Stroke Hemiplegic Patients: A Randomized Controlled Trial
}

\author{
Rajrupinder Kaur Rai, Lalit Arora, Smati Sambyal, Reena Arora \\ University College of Physiotherapy, Baba Farid University of Health Sciences, Faridkot, Punjab, India
}

\begin{abstract}
Background: Stroke is the second most common cause of death and major cause of long term disability worldwide. Several factors affect the functional independence in post stroke period in which trunk control and balance play a significant role. Despite evidence demonstrating the importance of trunk performance and balance after stroke, studies evaluating therapy aimed at improving trunk control, balance and gait are limited.
\end{abstract}

Aim: The aim of this study was to determine the effect of trunk rehabilitation and balance training on trunk control, balance and gait in post stroke patients.

Materials and methods: 30 patients based on inclusion and exclusion criteria were selected from the OPD of University College of Physiotherapy, Faridkot. Patients were equally divided into two groups based on randomization Group A $(n=15)$ and Group B $(n=15)$. Patients in Group A (Experimental Group) received trunk rehabilitation, balance training and conventional physiotherapy. Patients in Group B (Control Group) received conventional physiotherapy only. Duration of treatment was 5 weeks with treatment session for 4 days a week.

Outcome Measures: were Trunk Impairment Scale, Berg Balance Scale and 10 meters distance walk test.

Results: Both the groups showed improvements in trunk control, balance and gait of post stroke hemiplegic patients $(p<0.05)$. Between groups analysis showed that experimental group demonstrated more significant improvements in Trunk Impairment Scale, Berg Balance Scale scoring and the gait parameters i.e. gait speed and cadence post intervention.

Conclusion: Thus the study concluded that trunk rehabilitation exercises and balance training are effective in improving trunk control, balance and gait in post stroke hemiplegic patients.

Keywords: Stroke, Trunk control, Balance, Gait.

\section{Introduction}

Stroke is an acute onset of neurological dysfunction due to an abnormality in cerebral circulation with resultant signs and symptoms that corresponds to involvement of focal areas of the brain. WHO (1989) classified stroke as neurological deficit which must persist for at least 24 hour duration.Stroke is the most common life threatening neurological disease and is main cause of long term disability in adults worldwide. [12] Stroke is major health problem in India. The prevalence of stroke in India was estimated as 203 per 100,000 population above 20 years, amounting to a total of about 1 million cases. [3]

The effects of stroke are variable depending on location of the lesion as well as the size. The most typical symptom of stroke is hemiparesis or hemiplegia, which ranges from weakness to full paralysis of the body opposite to the side of the supratentorial lesion. [4]In addition to limb muscles trunk musculature is also impaired in stroke patients. Contrary to limb muscles in hemiplegia in which motor paralysis affects one side of the body the trunk muscles are impaired on both ipsilateral and contralateral side of body to that of lesion. Trunk muscles play an important role in the support of our bodies in antigravity postures such as sitting, standing and in the stabilization of proximal body parts during voluntary limb movements. [5] Good trunk stability is essential for balance and extremity use during daily functional activities. Several studies have identified deficits of trunk muscle strength and poor trunk control in stroke patients. [6] Poor recovery of trunk muscle performance results in a severe disability and a reduction in the activities of daily living. In stroke rehabilitation, trunk muscle performance is an important factor in predicting the functional outcome. [7]

In addition to limb and trunk impairments hemiplegic stroke patients frequently present balance abnormalities in relation to trunk impairment and are associated with poor balance and falls. [8]It has been demonstrated that stroke patients have abnormal and delayed postural responses in the lower extremity muscles in standing. Other postural control problems after stroke are loss of anticipatory activation of trunk muscles during voluntary movements, an increase in sway during quiet standing, a decreased area of stability in stance, delayed and disrupted equilibrium reactions, and reduced weight bearing on the paretic limb and increased risk of falling. [9] 
Trunk mobility impairment can cause gait, balance and postural problems because trunk control is related to measures of balance, gait and functional ability in patients with stroke.[10] Balance being the essential part of sitting, sit-to-stand and walking activities leads to increased risk of falling toward the paretic side is found to be significantly correlated with locomotor function and mobility reduced walking ability. [9] Thus slow walking after stroke may be a behavioral adaptation to poor endurance, poor balance and decreased stability. [2] Walking ability is closely associated with trunk stability. [11]

Most prior studies of performance after stroke are mainly concerned with the lower or upper extremity. In comparison with limb rehabilitation, trunk and balance recovery is a rather neglected area of stroke rehabilitation.

The altered trunk movements are a challenge for the maintenance of the body equilibrium, and restoration of normal movements of the trunk and of the pelvis in patients with stroke. Despite evidence demonstrating the importance of trunk performance after stroke, studies evaluating therapy aimed at improving trunk functions are limited.

Besides trunk rehabilitation exercises balance training is also essential for post stroke patients for recovery of balance and improvement in gait. Balance training is aimed at retraining of posturalcontrol, development of effective specificstrategies so that functional tasks can beperformed in changing environmental contexts and improving gait performance after stroke. Thus balance retraining is an important component of a comprehensive physicalrehabilitation programme.

Various studies have demonstrated the effects of therapeutical approaches used after stroke for example neurophysiologic, motor learning, strengthening exercises of limb muscles etc. But the evidence supporting the effectiveness of trunk rehabilitation and balance training is scarce. So this study was aimed at determining the effect of trunk rehabilitation and balance training on trunk control, balance and gait in post stroke patients.

\section{Material And Methods}

This ethical approval was granted by Research and Ethical committee of University College of Physiotherapy, This randomized controlled trial included a sample of 30 patients with post stroke hemiplegia. The study was conducted at the OPD of University College of Physiotherapy, Faridkot. The patients recruited in the study were referred from Department of Medicine and Neurosurgery, GGS Medical College and Hospital Faridkot. Informed consent was obtained from each participant.

The participants had to meet the following inclusion criteria: (1) Age 45-60 years, (2) both male and female patients, (3) first onset of unilateral stroke resulted in hemiplegia, (4) post stroke duration of at least 6 months, (5) able to understand and follow verbal commands, ability to stand with or without support, (6) scoring less than 21/23 on Trunk Impairment Scale, (7) scoring at least 30/56 0n Berg Balance Scale, (8) ability to walk independently at least $10 \mathrm{~m}$ distance, (9) currently not receiving any other type of therapeutic intervention.

The participants were excluded if they were diagnosed with stroke along with any other neurological disease affecting balance i.e. Parkinson's disease, Vestibular lesion and multiple sclerosis., impaired vision, had history of diagnosed musculoskeletal disorder of trunk and lower limbs affecting motor performance such as fracture, osteoarthritis, ligament injury in lower limb.

Patients were randomly assigned into two equal groups after initial evaluation. 15 patients assigned to Group A (experimental) and 15 to Group B (control).

\section{Intervention}

Patients in Group A (Experimental Group) received trunk rehabilitation, balance training and conventional physiotherapy. Patients in Group B (Control Group) received conventional physiotherapy only.Duration of treatment was 5 weeks with treatment session for 4 days a week.

Trunk rehabilitation exercises consisted of selective movements of upper and lower part of trunk in supine and sitting for 30 minutes.Supine exercises included pelvic bridging, unilateral pelvic bridging, upper trunk rotation by bringing clasped hands on either side, lower trunk rotation by moving knees on either side from crook lying, flexion rotation of upper trunkSitting exercises included the selective flexion extension of lower trunk, rotation of upper and lower trunk, forward and lateral reach. [5]

Balance training consisted of 20 minutes session which included following exercises: double leg stance for 10 seconds, tandem stance for 10 seconds, step forward and backward, step sideways on exercise step, sitting on a swiss ball with support and bending trunk forward and side to side, walking forward and backward in tandem walking pattern and perform single leg stance for 10seconds. [12]

Patients received conventional exercise program for 1 hour with repetition of 2 sets of 10 each. Exercises included upper extremity shoulder exercises: weight cuff and dumbbell exercises, hand exercises. Lower extremity exercise involved: sit to stand exercises, walking through obstacles, standing on a wobble board, partial squatting, toe rises. [13]

Patients in control group received conventional exercise program for 1 hour same as for Group A. 
Patients were assessed for trunk control, balance and gait. Data was collected at baseline of study (0 week) and at end of treatment (5 week). For assessment of trunk control Trunk Impairment Scale (TIS) was used, balance was evaluated by Berg Balance Scale (BBS), gait speed and cadence was calculated by 10 meter distance walk test.

\section{Data Analysis}

Data was analyzed using SPSS software. Paired and unpaired t tests were used to check the efficacy of trunk rehabilitation exercises, balance training and conventional physiotherapy.

Paired t test and unpaired t test was performed for variables Trunk Impairment Scale, Berg Balance Scale, Gait speed, cadence.

\section{Results}

Total 30 subjects participated in this study with age group of 45-60 years in both groups. $t$ test was performed to test significant difference in variables. The $\mathrm{p}=0.05$ was considered as level of significance. The mean age of subjects in group A was 52.93 and that of group B was 51.86.

Analysis was done between the pre test and post test values within group A and group B to determine the changes within the variables Trunk impairment scale (TIS), Berg balance scale (BBS), gait speed and cadence for trunk control, balance and gait. There was significant improvements in scoring of Trunk impairment scale (TIS), Berg balance scale (BBS), gait speed and cadence with $\mathrm{p}$ value $<0.05$. The results showed that there were improvements in trunk control, balance and gait parameters after intervention in post stroke patients.

Comparison between the TIS score of both the groups has been done. There was not much difference in TIS score between pre values of both the groups with mean values of 16.200 and 14.733 , but in post values of TIS, there was a significant difference in the TIS score between both groups $(\mathrm{p}<0.05)$. Group A shows highly significant improvement than Group B in TIS score with mean value of 21.333 (Group A) and 15.400 (Group B) after 5 weeks of treatment.

BBS score of both the groups was compared. There was no significant difference in Berg balance scale between pre values of both the groups with $p>0.05$, but in post test values there was a significant difference in BBS scoring between both the groups $(\mathrm{P}<0.05)$. Group A shows highly significant improvement than Group B in BBS score with mean value of 53.000 (Group A) and 45.133 (Group B) after treatment.

Similarly Comparison between the gait speed based on $10 \mathrm{~m}$ walk distance test of both the groups was done. There was no significant difference in pre values of gait speed between the groups with $\mathrm{p}>0.05$, but in post test values there was a significant difference in between both the groups $(\mathrm{P}<0.05)$. Group A shows highly significant improvement than Group B in gait speed with mean value of 0.8907 (Group A) and 0.5580 (Group B) after treatment.

Comparison of cadence between both the groups based on $10 \mathrm{~m}$ walk distance was done. There was no significant difference in pre values of cadence between the groups with $p>0.05$, but in post test values there was a significant difference in between both the groups $(\mathrm{P}<0.05)$. Group A shows highly significant improvement than Group B in cadence with mean value of 88.640 (Group A) and 73.213 (Group B) after treatment.

Group A and group B were compared to check changes within groups in trunk control, balance, gait speed and cadence with variables trunk impairment scale, berg balance scale, gait speed and cadence.

Group A shows highly significant improvement than group B in TIS with mean value of 5.133 (Group A), 0.6667 (Group B), BBS with mean value of 7.60 (Group A), 0.80 (Group B), Gait speed with mean value of 0.3700 (Group A), 0.033 (Group B) and cadence with mean value of 21.040 (Group A), 7.313 (Group B).

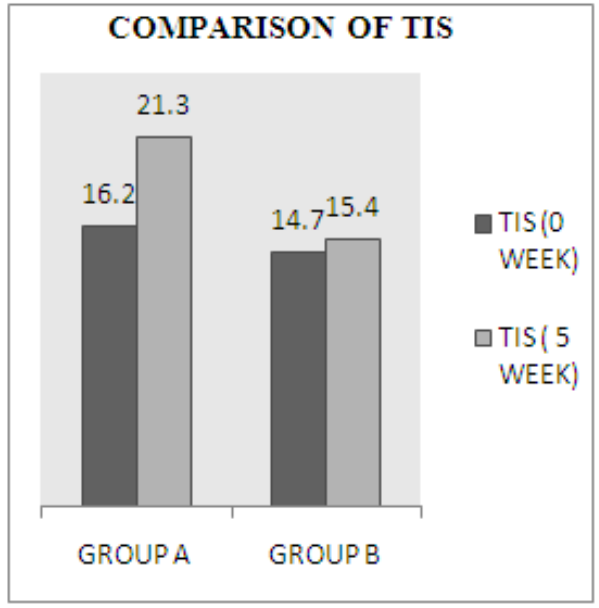

Fig. 1 Intra group comparison of TIS

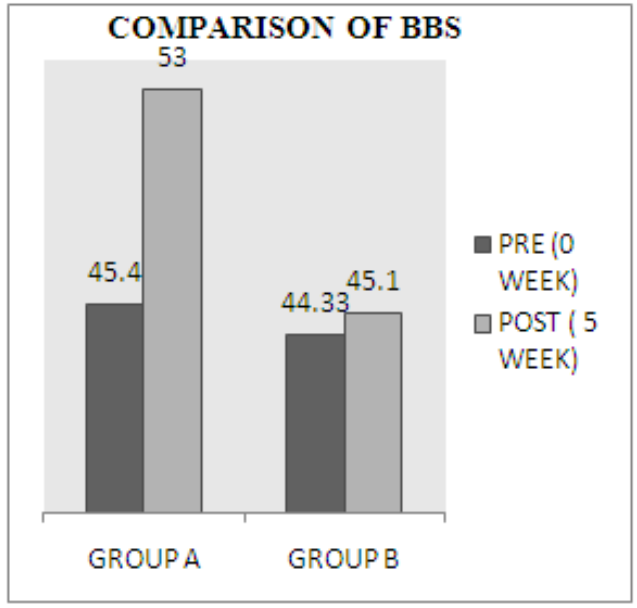

Fig. 2 Intra group comparison of BBS 


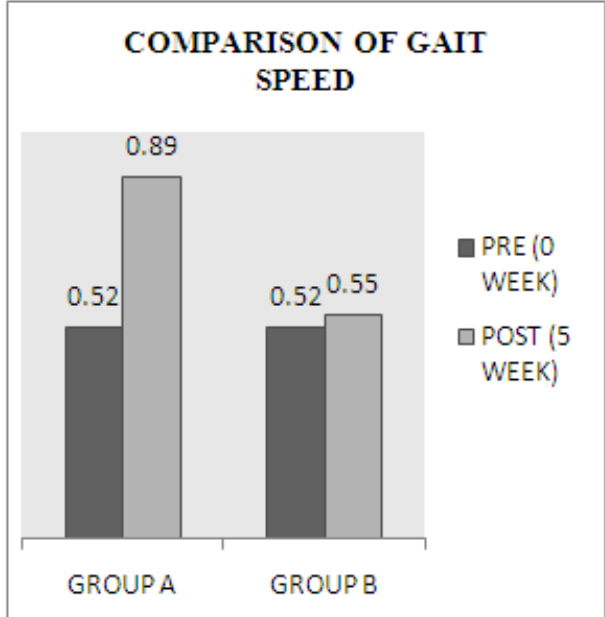

Fig. 3 Intra group comparison of Gait speed

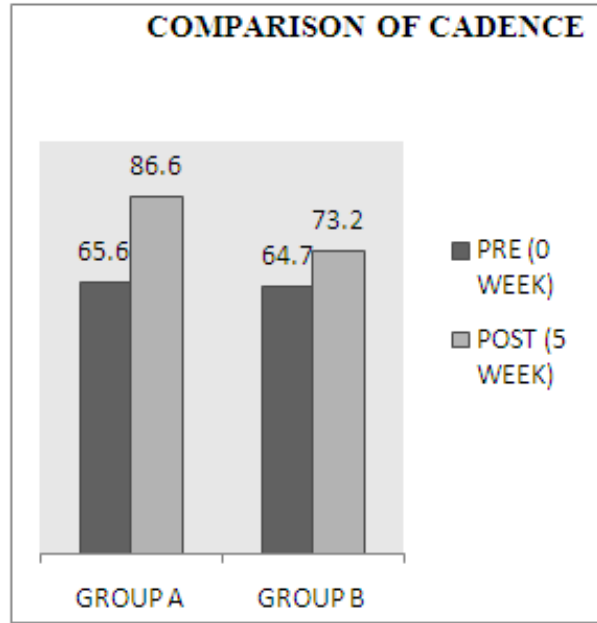

Fig. 4Intra group comparison of Cadence

\section{Discussion}

The findings of this study suggest that trunk rehabilitation and balance training in addition with conventional physiotherapy are better than conventional physiotherapy alone in improving trunk control, balance and gait. This study shows that trunk exercises and balance training was effective at enhancing trunk control and balance but gait also improved significantly. The results of present study are consistent with other studies which concluded the effect of trunk rehabilitation and balance training on measures of trunk control, balance and gait.

A study by Karthikbabu S (2011) supports present study. He studied the role of trunk rehabilitation on trunk control, balance and gait in patients with chronic stroke. The study showed large effect size for Trunk impairment scale (1.75), Berg balance scale (1.65) than for gait parameters (0.65). After trunk rehabilitation there was significant improvement in cadence, gait speed. The study indicated the importance of trunk exercises in the rehabilitation of stroke patients. [5]

In relevance to results of balance training the study by Dean (2000) found to be supportive to present study. In his research he studied the effect of task specific balance training on rate of falls post stroke. Balance exercises found to be effective in improving balance and effect is also carried over to gait. The overall results of his study concluded that balance training improves the balance and walking speed of stroke patients. [14]

The findings of the present study indicate the use of trunk rehabilitation exercises and Balance training in addition to a conventional stroke rehabilitation program is beneficial in improving trunk control, balance and gait parameters of chronic post stroke hemiplegic patients.

The improvements in trunk control may be due to the fact that the trunk exercises mainly consisted of selective trunk movements which helped in strengthening of trunk muscles and also increased awareness of trunk position and anticipatory postural adjustments which also helped in achieving good trunk control.

Improvements in balance and gait occurred because both the trunk rehabilitation programme and balance training consist of the use of lower limb muscles which account in change of balance and gait. Gait improved just not because of selective flexion and extension movements but also because of rotation exercises of upper and lower trunk. Gait and balance also improved because the motor control proceeds from proximal to distal, the improved level of proximal trunk control leads to improvement in distal lower limb control which helped in attaining better balance and gait.

\section{Conclusion}

This study concluded that trunk rehabilitation and balance training is found to be effective in improving trunk control, balance and gait. Improvement in trunk control, balance and gait occurred to a greater extent after the treatment of five weeks in subjects who received trunk rehabilitation, balance training and conventional physiotherapy as compared to patients who received only conventional physiotherapy. The results of study support the importance of trunk rehabilitation exercises and balance training in the rehabilitation of hemiplegic patients after stroke.

Some limitations were identified in the study such as the small sample size for the study challenges the generalizability of the study, follow up assessment was not performed, the study did not investigate the long term effects of trunk and balance exercises. 
Thus future studies need to be done with other outcome measures such as posturography and electromyographic analysis and biofeedback, large sample size should be taken.Long term follow up studies should be done to know the carry over effects of intervention.

\section{References}

[1]. World Health Organization. Task Force on Stroke and other Cerebrovascular Disorders: Recommendations on stroke prevention, diagnosis and therapy. Stroke.1989; 20(10): 1407-1431.

[2]. Vats M 2013, 'Efficacy of task specific step-up exercises on the gait Parameters of chronic hemiparetic stroke individuals' International Journal of Physiotherapy and Research, Vol. 1,pp.30-37.

[3]. Sethi PK 2002, 'Incidence in India and management of ischaemic stroke', Neurosciences today, vol. 4, pp. 139-143.

[4]. O’ Sullivan SB \&Schimtz 200, Physical Rehabilitation assessment and treatment, $4^{\text {th }}$ edn.,F.A.Davis, Philadelphia,pp. 519- 581.

[5]. Karthikbabu S, Rao BK, Manikandan N, Solomom JM \&Chakrapani M 2011, 'Role Of Trunk Rehabilitation On Trunk Control, Balance And Gait In Patients With Chronic Stroke, A Pre Post Design', Neurosci Med, vol. 2, pp.61-67.

[6]. Nayak A, Vijayakumar K, Misri ZK\&Karthikbabu S 2012, 'Does training on swiss ball improve trunk performance after stroke? A single blinded, quasi experimental study design', vol. 6, pp. 172-175.

[7]. Fujiwara T, Sonoda S, Okajima Y \& Chino N 2001, 'The relationships between trunk function and the findings Of transcranial magnetic stimulation among patients with Stroke', J Rehabil Med, vol. 33, pp. 249-255.

[8]. Jijimol G, Fayaz RK \&Vijesh PV 2013, 'Correlation of trunk impairment with balance in patients with chronic stroke', Neurorahabilitation, vol. 32, pp.321-325.

[9]. Yavuzer G, Eser F, Karakus D, Karaoglan B \&Stam HJ 2006, ' The Effects of balance training on gait late after stroke : a randomised controlled trial', Clinical Rehabilitation, vol. 20, pp. 960-969.

[10]. Reyerson S, Byl NN, Brown DA, Wong RA \&Hidler JM 2008, 'Altered Trunk Position Sense And Its Relation To Balance Functions In People After Stroke', J NeurolPhysTher, vol. 32, pp.14-20.

[11]. Yue LAN, Guang-qing XU, Dong-feng H, Yu-rong M, Shao-zhen C, Zhong PEI \& Jin-Sheng Z 2013, 'Association between improved trunk stability and walking capacity using ankle-foot orthosis in hemiparetic patients with stroke: evidence from threedimensional gait analysis', Chinese Medical Journal, vol. 126, pp. 3869-3873.

[12]. Bayouk JF, Boucher JP \&Leroux A 2006, 'Balance Training Following Stroke: Effects of Task Oriented Exercises with or without Altered Sensory Input',Int J RehabilRes, vol.29, pp. 51-59.

[13]. Pang MY, Harris JE, Eng J \& 2006, 'A community-based upper-extremity group exercise programme improves motor function and performance of functional activities in chronic stroke: A randomized controlled Clinical Trial', Arch phys med rehabil.,vol.87, pp. $1-9$.

[14]. Dean CM, Richards CL \&Malouin F 2000, 'Task - relation circuit training improves performance of locomotor tasks in chronic stroke: A Randomised, Controlled pilot trial' Arch PhysMed Rehabilitation, vol. 81, pp. 409-416. 